\title{
Identification of microRNA signature in the progression of gestational trophoblastic disease
}

\author{
Jiu-Ru Zhao ${ }^{1,2}$, Wei-Wei Cheng ${ }^{3}$, Ya-Xin Wang ${ }^{1,4}$, Mei Cai ${ }^{1,2}$, Wei-Bin Wu ${ }^{1,2}$ and Hui-Juan Zhang ${ }^{1,2}$
}

\begin{abstract}
Gestational trophoblastic disease (GTD) encompasses a range of trophoblast-derived disorders. The most common type of GTD is hydatidiform mole (HM). Some of HMs can further develop into malignant gestational trophoblastic neoplasia (GTN). Aberrant expression of microRNA (miRNA) is widely reported to be involved in the initiation and progression of cancers. MiRNA expression profile also has been proved to be the useful signature for diagnosis, staging, prognosis, and response to chemotherapy. Till now, the profile of miRNA in the progression of GTD has not been determined. In this study, a total of 34 GTN and 60 complete HMs (CHM) trophoblastic tissues were collected. By miRNA array screening and qRT-PCR validating, six miRNAs, including miR-370-3p, -371a-5p, -518a-3p, -519d-3p, -520a$3 p$, and -934 , were identified to be differentially expressed in GTN vs. CHM. Functional analyses further proved that miR-371a-5p and miR-518a-3p promoted proliferation, migration, and invasion of choriocarcinoma cells. Moreover, we demonstrated that miR-371a-5p was negatively related to protein levels of its predictive target genes BCCIP, SOX2, and $B N I P 3 L$, while miR-518a-3p was negatively related to MST1 and EFNA4. For the first time, we proved that miR-371a-5p and miR-518a-3p directly targeted to 3'-UTR regions of BCCIP and MST1, respectively. Additionally, we found that miR$371 a-5 p$ and miR-518a-3p regulated diverse pathways related to tumorigenesis and metastasis in choriocarcinoma cells. The results presented here may offer new clues to the progression of GTD and may provide diagnostic biomarkers for GTN.
\end{abstract}

\section{Introduction}

Gestational trophoblastic disease (GTD) is a group of diseases originating from trophoblastic cells with abnormal proliferation and metastasis. GTD heterogeneously comprises gestational trophoblastic neoplasia (GTN), hydatidiform mole (HM), benign non-neoplastic lesions, and villous lesion ${ }^{1,2}$. HM, the most common member of GTD, includes two entities: partial hydatidiform mole (PHM) and complete hydatidiform mole (CHM). PHM is

\footnotetext{
Correspondence: H-J. Zhang (zhanghj815@126.com)

'Departments of Pathology and Bio-Bank, International Peace Maternity and Child Health Hospital, Shanghai Jiao Tong University School of Medicine, Shanghai 200030, China

${ }^{2}$ Institute of Embryo-Fetal Original Adult Disease Shanghai Jiao Tong University School of Medicine, Shanghai 200030, China

Full list of author information is available at the end of the article Edited by G. Calin
}

characterized by partial edema and focal trophoblastic proliferation in chorionic villi, while $\mathrm{CHM}$ presents exaggerated proliferation of abnormal trophoblast in nearly every villus. PHM is genetically almost triploid with one maternal chromosome set and two paternal chromosome sets while CHM is generally androgenetic diploid $^{3}$. Incidence of GTD could reach up to 2.0 per 1000 pregnancies ${ }^{4}$. Generally, most HMs would regress after uterine evacuation, whereas about 15\%-20\% of CHM cases and $0.5 \%$ of PHM cases could progress into $\mathrm{GTN}^{5,6}$. Clinically, persistently raised or rising serum human chorionic gonadotropin (hCG) level after evacuation of $\mathrm{HM}$ is an indication of GTN. Although GTN patients showed more than $90 \%$ cure rate with a good response to chemotherapy, around $4 \%$ cases would succumb to chemotherapy ${ }^{7}$. Currently, the exact molecular mechanisms 
of etiopathogenesis and progression of GTD still remain largely unknown.

MicroRNAs (miRNAs) are 19-24 nucleotides small noncoding RNAs that participate in all fundamental cellular processes, involving in the regulation of translation and degradation of nearly $50 \%$ of the human mRNAs ${ }^{8}$. Aberrant expression of miRNAs has been widely detected in human cancers ${ }^{9}$. For example, Iorio et al. identified 29 dysregulated miRNAs in breast cancer ${ }^{10}$, Lee et al. identified 112 aberrantly expressed miRNAs in pancreatic cancer ${ }^{11}$, and Yanaihara et al. discovered 43 differentially expressed miRNAs in lung cancer ${ }^{12}$. Besides, increasing evidences indicated that miRNAs dysregulation could be an early event that occurred in precancerous stage ${ }^{13}$. Many miRNAs have been reported to be signatures of cancer prognosis, such as miR-15a and miR-16-1 in chronic lymphocytic leukemia, miR-143 and miR-145 in colorectal cancer, let-7 in lung cancer, miR-155 in diffuse large B-cell lymphoma, miR-221 and miR-222 in papillary thyroid carcinoma, and miR-375 in laryngocarcinoma ${ }^{14}$. Moreover, miRNAs have been proved to be a more accurate marker for the classification of cancer subtype than mRNAs, and several miRNAs are common signatures for different cancers. For example, miR-21 was reported to be overexpressed in six types of cancers, and miR-17-5p and miR-191 were overexpressed in five ${ }^{15}$.

So far, genome-wide analysis has identified more than 2500 human miRNAs, including over 700 miRNAs in human placenta. Several miRNAs are trophoblast-specific, such as the most intriguing family-chromosome 19 miRNA cluster (C19MC), which contains 46 intronic miRNA genes that are scattered over $100 \mathrm{~kb}$ of genomic DNA and produces 58 mature miRNAs ${ }^{16}$. Although numerous progresses in placenta-associated miRNAs have been made, studies about miRNAs in the pathogenesis and progression of GTD are still rudimentary. Previously, Na et al. reported that miR-517a, $-517 b,-518 b$, and $-519 a$ were dysregulated in $\mathrm{CHM}^{17}$. Hasegawa et al. found high levels of miR-520b, -520f, and -520c-3p significantly decreased in plasma of CHM patients after evacuation ${ }^{18}$. Miura et al. further demonstrated that these three miRNAs showed similar variation tendency to serum hCG concentration in GTN patients ${ }^{19}$. Chao et al. discovered that miR-199b was downregulated in human choriocarcinoma cells compared with normal trophoblasts ${ }^{20}$. Previously, we also discovered that miR-21 was overexpressed in $\mathrm{GTN}^{21}$. However, most of these studies were based on very small sample sizes, making these results less conclusive. Systemic investigation about the expression profile and roles of miRNAs in GTD is still needed.

In the present study, we aimed to explore the differentially expressed miRNAs in GTN vs. CHM. Further, we sought to determine the roles of dysregulated miRNAs in proliferation and metastasis of trophoblastic cells, and to elucidate the molecular regulation networks. Our results would be helpful for the investigation of early molecular biomarkers and therapeutic targets for GTN.

\section{Results \\ Altered miRNA expression profile in GTN vs. CHM}

A small-scale miRNA microarray was performed to evaluate miRNA expression profiles in GTN, CHM, and normal first-trimester placentas. The ANOVA and $t$-tests were used to identify miRNAs whose expression were significantly different among these three different categories of trophoblastic tissues based on normalized data ${ }^{10}$. Totally, 119 upregulated and 134 downregulated miRNAs were identified $(P<0.05)$ in GTN tissues compared with CHM. Meanwhile, cluster analysis generated a tree with clear distinction between GTN and normal first-trimester placentas (Supplementary Figure S1a). Scatter plot analysis further indicated that there were several differentially expressed miRNAs between GTN and CHM (Supplementary Figure S1b). Sixteen miRNAs were found with over fivefold changes between GTN and CHM, including miR10b-5p, -21, -98-5p, -99a-5p, -125b-2-3p, -196b-5p, -196b3p, -370-3p, -371a-5p, -372-3p, -518a-3p, -518b, -519d-3p, $-520 a-3 p,-584-5 p$, and -934 . For validation, quantitative real-time polymerase chain reaction (qRT-PCR) test for these 16 miRNAs was carried out in fresh tissues (13 cases of GTN, 25 cases of CHM) and in formalin-fixed, paraffinembedded (FFPE) tissues (21 cases of GTN, 35 cases of CHM). Notably, six miRNAs, including miR-370-3p, -371a5p, -518a-3p, -519d-3p, -520a-3p, and -934, were identified to be differentially expressed in GTN vs. CHM (Fig. 1a). Among which, all, except miR-370-3p, were upregulated in GTN. Besides, relative expression levels of miR-371a-5p and miR-518a-3p, the two most differentially expressed miRNAs, were also detected in choriocarcinoma cells. Similar to the expression pattern in GTN, levels of both miRNAs were at least fivefolds higher in choriocarcinoma cells than in normal primary human trophoblastic cells (PHT) and HTR-8/SVneo cells (Fig. 1b, all $P<0.001$ ). To address the cellular origin of miR-371a-5p and miR-518a$3 p$ in trophoblastic tissues, in situ hybridization (ISH) was performed in the 56 FFPE tissues. As indicated in Fig. 1c, positive signals were detected predominantly in trophoblastic layers for both miRNAs, and were much stronger in GTN than in CHM. NBT/BCIP staining also revealed that the staining scores of both miRNAs were significantly higher in GTN than in CHM (Supplementary Figure S2, Tables S1 and S2). Considered together, our data indicated that miR-371a-5p and miR-518a-3p were upregulated in villous trophoblastic cells of GTN.

\section{MiR-371a-5p and miR-518a-3p promoted proliferation of choriocarcinoma cells}

To investigate the functions of miR-371a-5p and miR518a-3p in choriocarcinoma cells, firstly, BeWo, JAR, and 


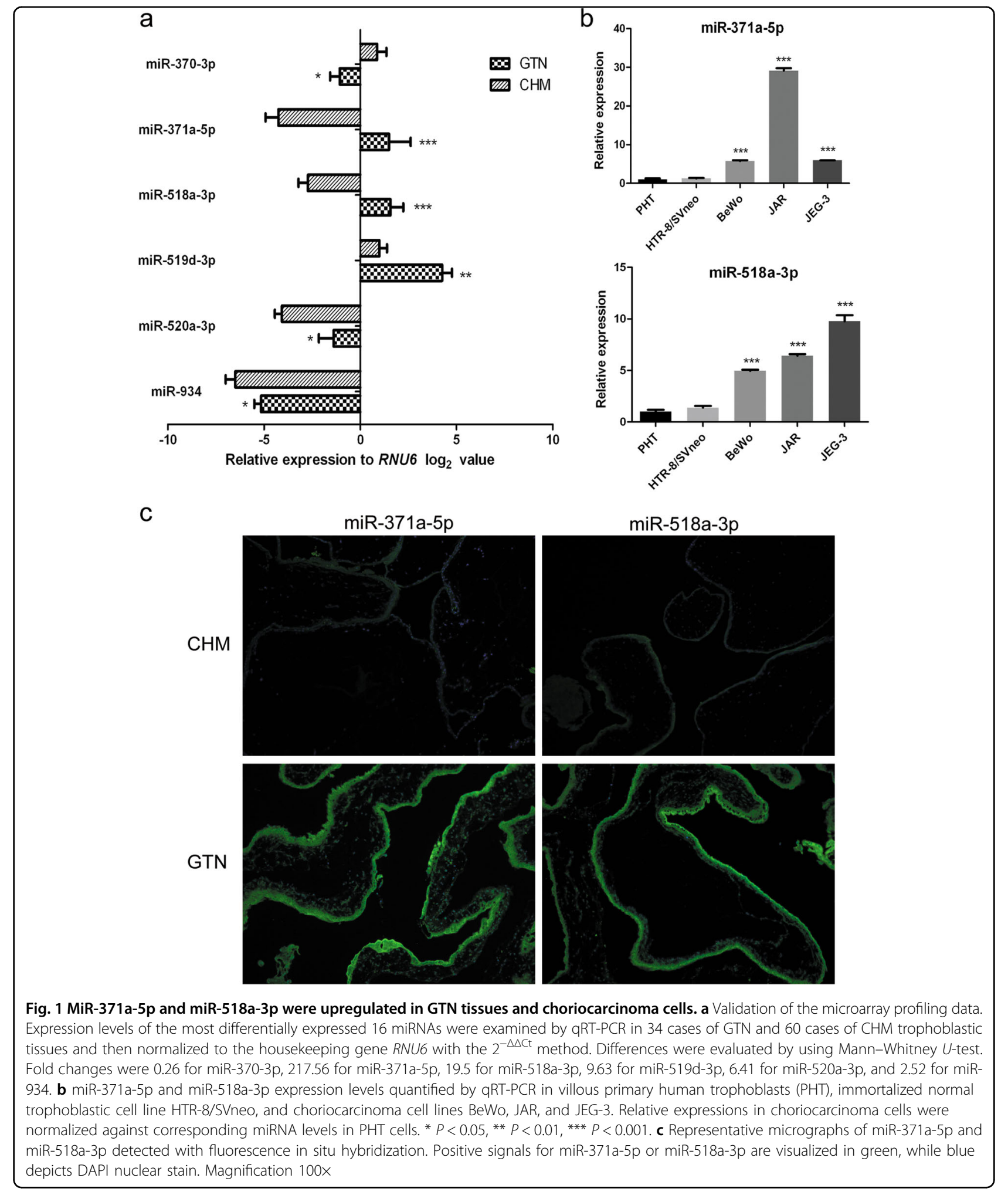

JEG-3 cells were transiently transfected with corresponding miRNA mimics, inhibitors, or controls. qRT-PCR results indicated that overexpressions of miR-371a-5p and miR$518 a-3 p$ induced at least 50-folds and 150-folds increases in all three cell lines, respectively (Supplementary Figure S3a), while silencing of both miRNAs reduced their expression by more than $60 \%$ in all three cell lines (Supplementary Figure S3b). Then, cell viabilities were measured by CCK- 8 


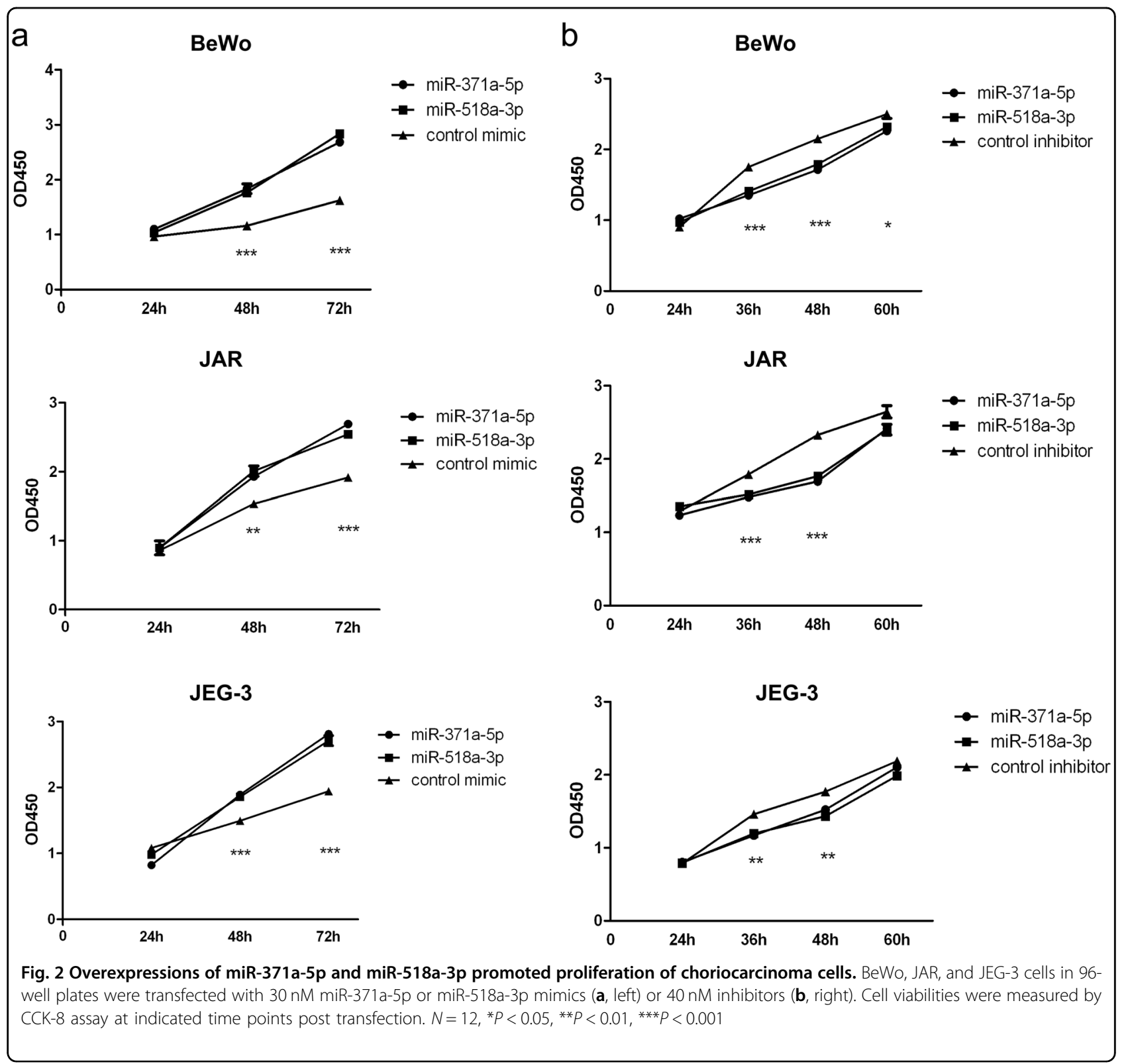

assay at different time points post transfection (Fig. 2). Results indicated that overexpression of miR-371a-5p or miR-518a-3p significantly promoted proliferation of BeWo, JAR, and JEG-3 cells, whereas downregulation of miR371a-5p or miR-518a-3p significantly inhibited proliferation of the three choriocarcinoma cells.

\section{Overexpression of $\mathrm{miR}-371 \mathrm{a}-5 \mathrm{p}$ or $\mathrm{miR}-518 \mathrm{a}-3 \mathrm{p}$ reduced} the percentage of choriocarcinoma cells at the $S$ phase of cell cycle

Forty-eight hours post transfection with mimics or inhibitors of miR-371a-5p and miR-518a-3p, the percentages of choriocarcinoma cells at each cell cycle phase were analyzed. Flow cytometric analysis (FCA) indicated that cells at the $\mathrm{S}$ phase were significantly reduced in all three choriocarcinoma cells with overexpressions of miR-371a$5 \mathrm{p}$ (0.66-fold in BeWo, 0.85-fold in JAR, 0.73-fold in JEG-3) or miR-518a-3p (0.82-fold in BeWo, 0.92-fold in JAR, 0.81fold in JEG-3) (all $P<0.01$ ). An increase in the percentages of cells at the G1 and G2/M phases was also observed in BeWo and JEG-3 cells post transfection with miR-371a-5p or miR-518a-3p mimics (Fig. 3a). In contrast, knockdown of both miRNAs arrested cell cycle at the $\mathrm{S}$ phase in all three cells (about 1.20-fold increase for miR-371a-5p and 1.1-fold increase for miR-518a-3p, both $P<0.05$ ), thus hindered the entry to cell replication cycle (Fig. 3b). 
MiR-371a-5p and miR-518a-3p promoted migration and invasion of choriocarcinoma cells

Transwell assay was adopted to evaluate the effect of miR-371a-5p and miR-518a-3p on migration and invasion of choriocarcinoma cells. To eliminate the potential confounding effect of cell proliferation, mitomycin $\mathrm{C}$ was used $^{22}$. Cell-number counting and CCK- 8 assay revealed that $2 \mathrm{~h}$ pretreatment with $8 \mu \mathrm{g} / \mathrm{ml}$ mitomycin C for JEG3 cells or $4 \mu \mathrm{g} / \mathrm{ml}$ mitomycin C for BeWo and JAR cells could block their proliferation within $36 \mathrm{~h}$ (data not shown). Thus, cells were pretreated with mitomycin $\mathrm{C}$ for $2 \mathrm{~h}$ before transwell assay. Marked increases in the number of migrated or invaded cells were observed in the miR-371a-5p or miR-518a-3p overexpressed groups (Fig. 4a and Supplementary Figure S4, all >2-folds, $P<0.001)$. Conversely, significant decreases in the number of migrated or invaded cells were detected in the
miR-371a-5p or miR-518a-3p knockdown groups (Fig. 4b and Supplementary Figure S5).

\section{Validation of miR-371a-5p and miR-518a-3p potential targets}

Given that miRNA function generally relies on their target genes, to further investigate regulation networks of miR-371a-5p and miR-518a-3p, their putative targets were predicted by TargetScan (http://www.targetscan. org), PicTar (http://pictar.mdc-berlin.de), miRanda (http://www.microrna.org), and miRecords (http://c1. accurascience.com/miRecords/). Based on enrichment analysis in Gene Ontology (GO) database, anti-oncogenes or genes encoding proteins with potential tumorsuppressing functions were figured out. BCCIP, BNIP $3 L$, and $S O X 2$ were selected as representatives of miR-371a5p downstream targets, while MST1 and EFNA4 were

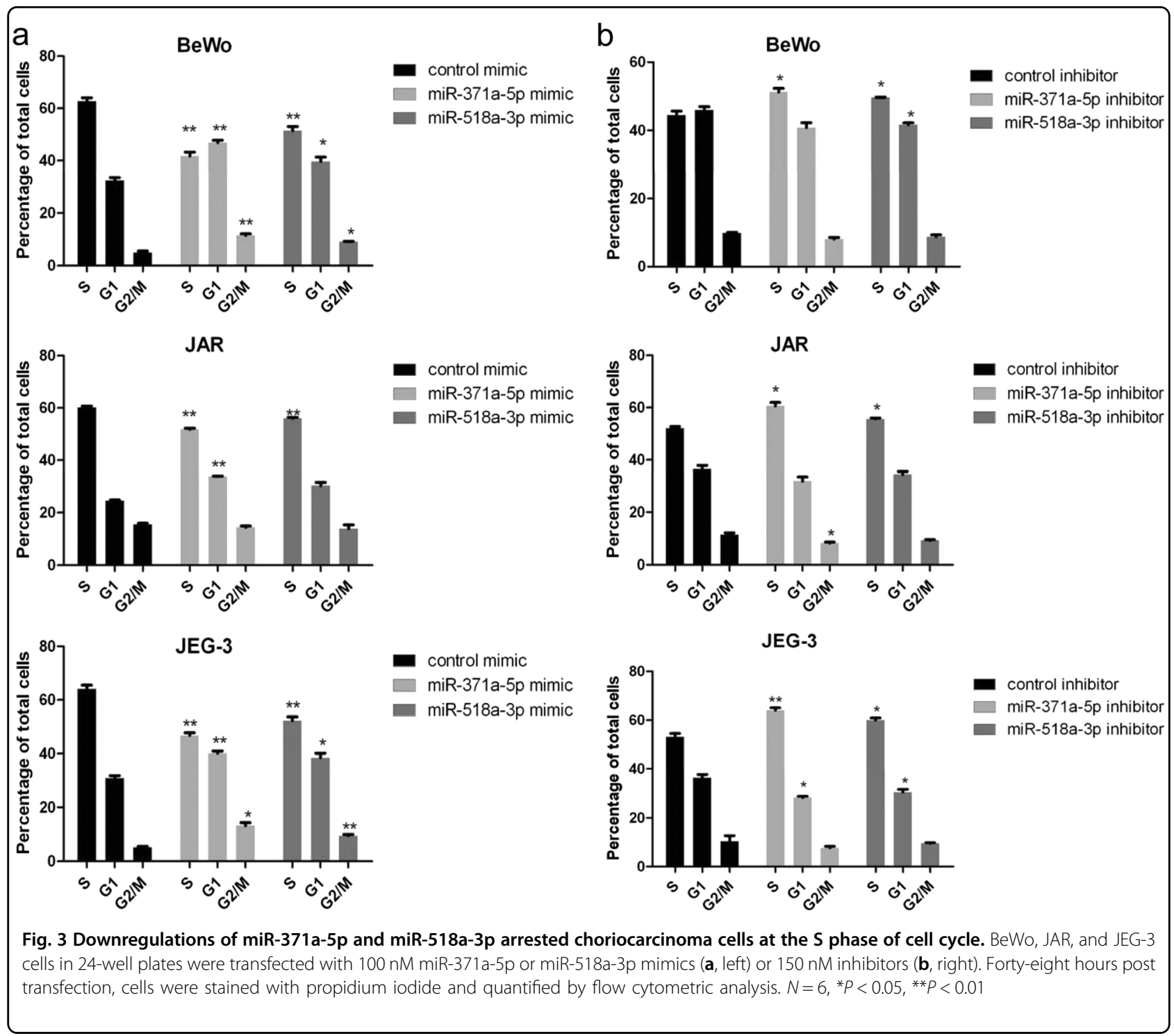




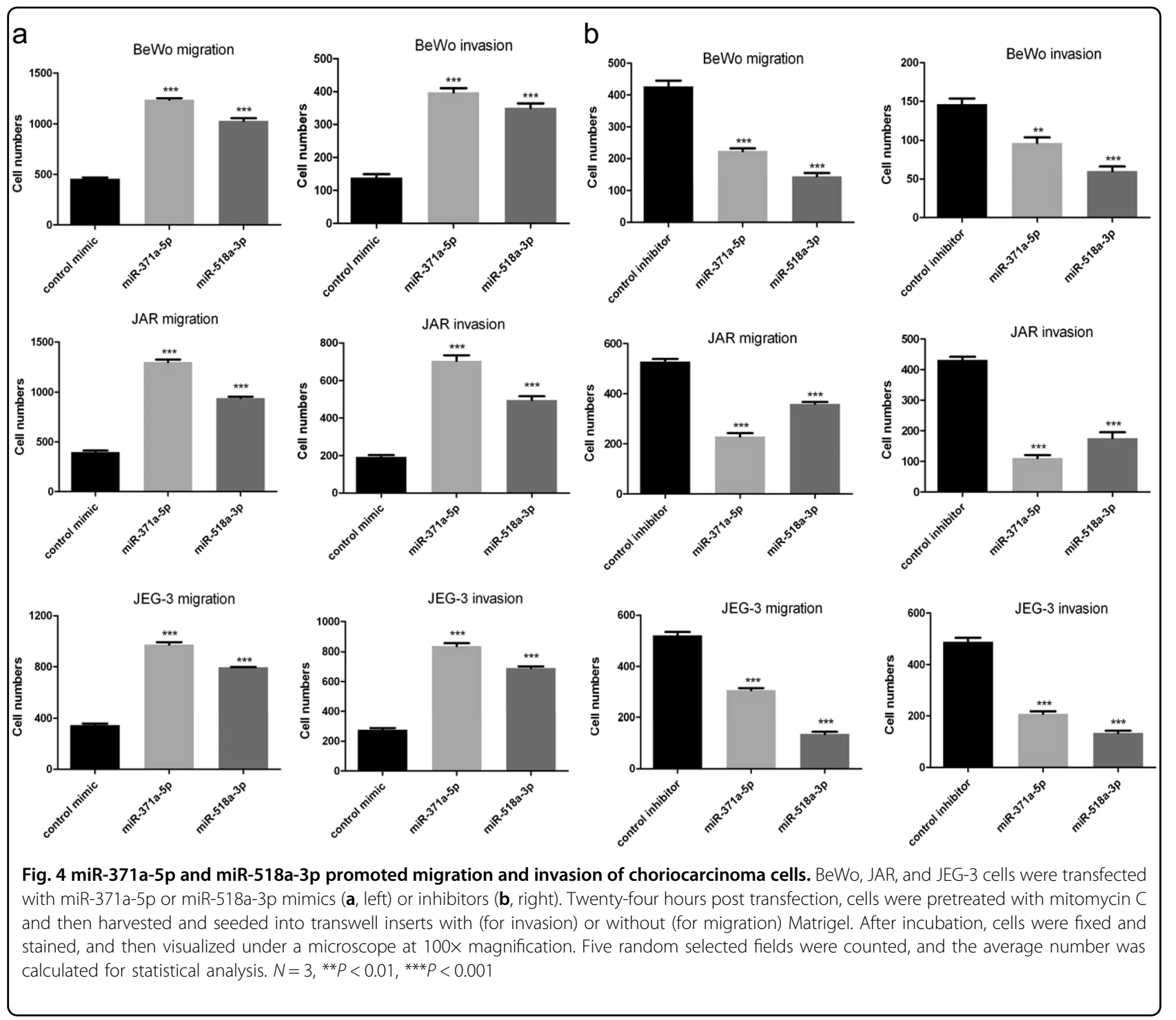

selected as representatives of miR-518a-3p downstream targets. BeWo, JAR, and JEG-3 cells were harvested at 36 $\mathrm{h}$ post miRNA mimics or inhibitors transfection. Then, qRT-PCR assay was carried out to investigate the alteration of transcripts of these genes. As indicated in Figs. 5a and $6 \mathrm{a}$, overexpression of both miRNAs did not induce any significant change in these genes at mRNA level even compared with the knockdown groups. Meanwhile, western blotting demonstrated significantly decreased protein levels of BCCIP, SOX2, and MST1 in corresponding miRNAs overexpressed group, whereas exhibited a remarkable augmentation of these proteins in knockdown groups. However, the level of BNIP3L was rather low in all three cell lines, and only obvious elevated expression was observed in miR-371a-5p knockdown JAR cells. Besides, expression of EFNA4 was also low in BeWo and JAR cells, while significant alterations were only detected in miR- 518a-3p knockdown JAR and JEG-3 cells (Figs. 5b and $6 b)$. To verify the direct relationships of miR-371a-5p and miR-518a-3p with their potential target genes, wild-type and mutated 3 '-UTR regions of BCCIP and MST1 were constructed to a report vector as the representatives. To minimize the background, HTR-8/SVneo cells were used since the endogenous expressions of miR-371a-5p and miR-518a-3p were much lower than choriocarcinoma cells (Fig. 1b). Transient co-transfection with the wildtype $3^{\prime}$-UTR reporter plasmid BCCIP-WT and miR-371a$5 \mathrm{p}$ mimic led to a significant decrease in reporter activity (Fig. 5c). Meanwhile, significantly increased reporter activity was detected when BCCIP-WT co-transfected with miR-371a-5p inhibitor as compared with the control. Besides, reporter activities were almost unaffected when cells were co-transfected with the mutated 3'-UTR reporter plasmid BCCIP-MUT and miR-371a-5p 
a
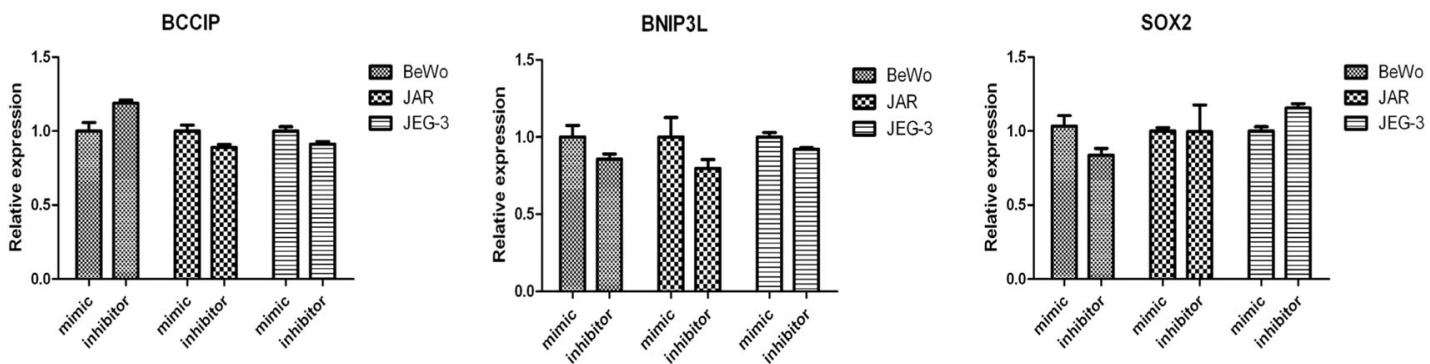

b

BCCIP
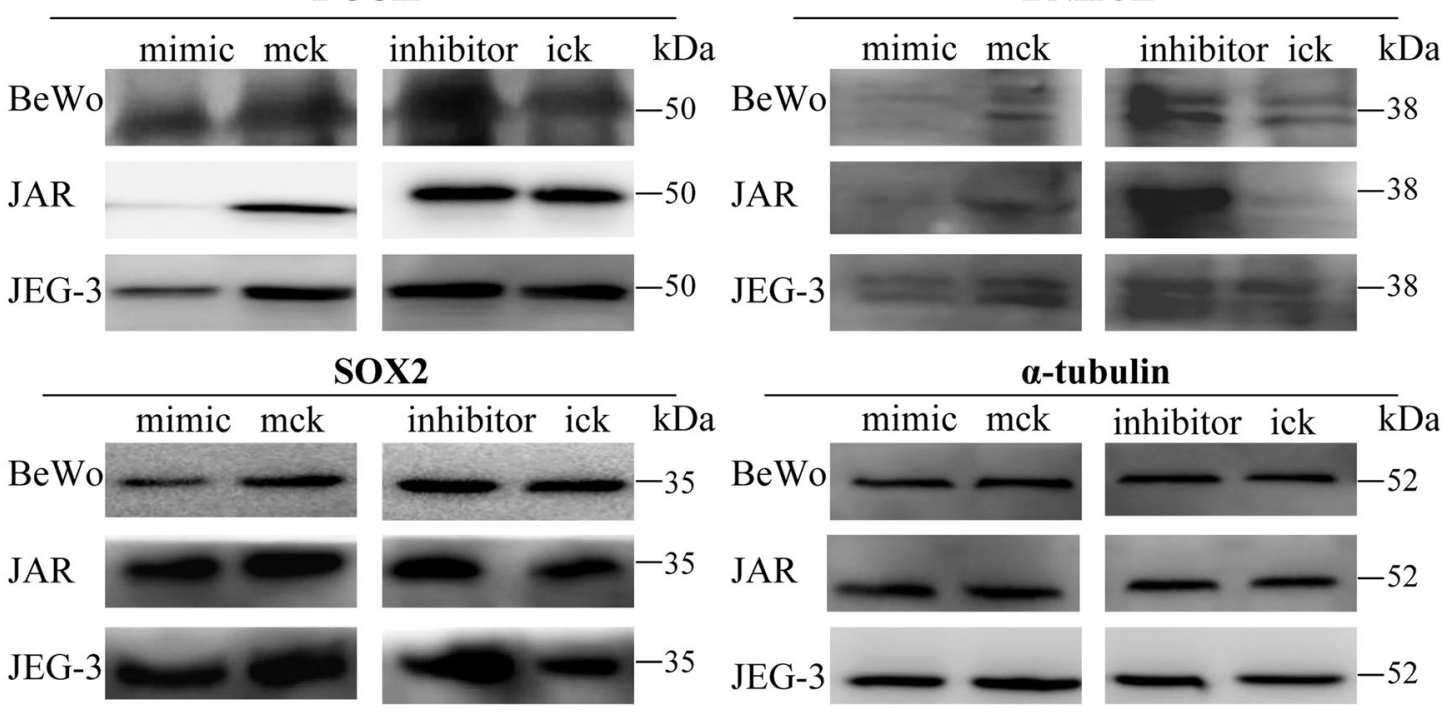

a-tubulin

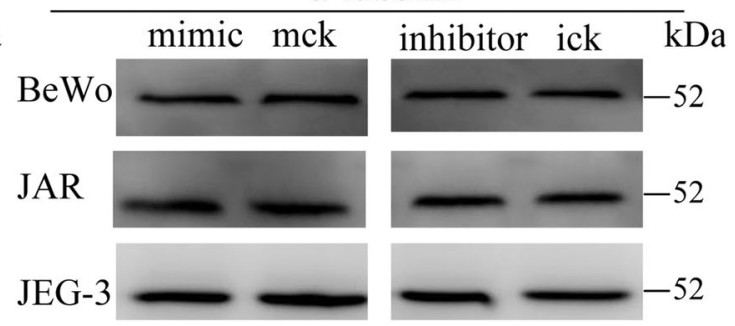

$\mathrm{C}$

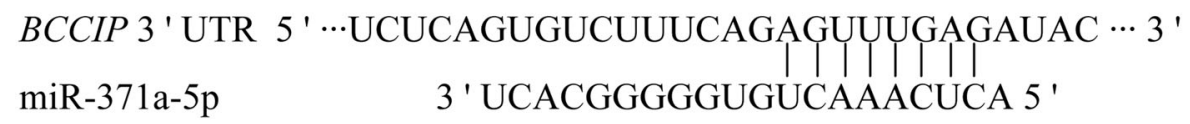

Mutant 3 ' UTR 5 ' …UCUCAGUGUCUUUCAGUUAAGCUCAUAC $\cdots 3$ '
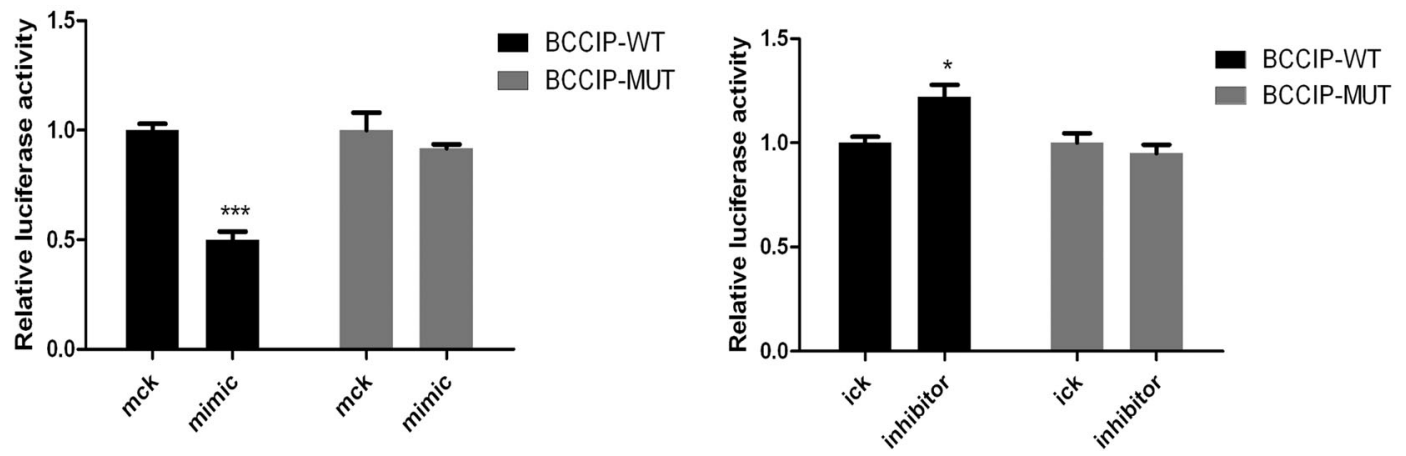

Fig. 5 Validation of miR-371a-5p potential target genes $B C C I P, B N I P 3 L$, and SOX2. a qRT-PCR quantification of the mRNA levels of BCCIP, BNIP3L, and SOX2 genes. BeWo, JAR, and JEG-3 cells were transfected with miR-371a-5p mimic/inhibitor or control mimic/inhibitor. Cells were harvested at $36 \mathrm{~h}$ post transfection, and $a$-tubulin was used as the endogenous reference gene. $\mathbf{b}$ Western blotting analysis of BCCIP, BNIP3L, and SOX2. Cells were harvested at $48 \mathrm{~h}$ post transfection, and a-tubulin was used as the endogenous control. c Direct interaction of miR-371a-5p and BCCIP. Upper: bioinformatic predicted miR-371a-5p targeting site in 3'-UTR of BCCIP. Lower: luciferase activity assay. At $48 \mathrm{~h}$ post co-transfection with BCCIP-WT or BCCIP-MUT and miR-371a-5p mimic/inhibitor or control mimic/inhibitor, luciferase activities were measured in HTR-8/SVneo cells. Renilla luciferase activities in corresponding controls were normalized to 1 . Mck stands for control mimic and ick stands for control inhibitor. $N=9,{ }^{*} P<0.05,{ }^{* * *} P<$ 0.001 
a

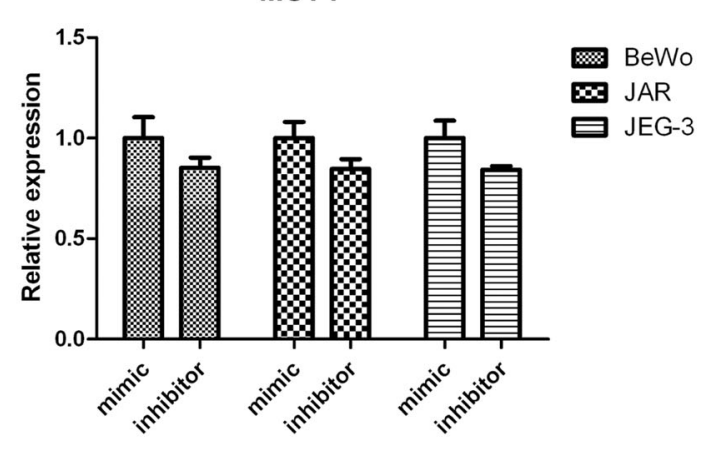

b

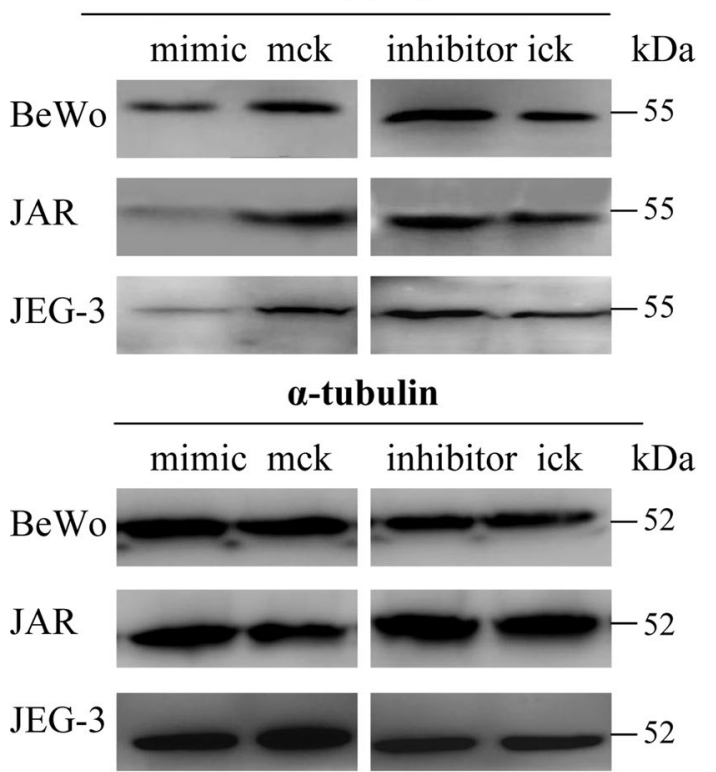

EFNA4

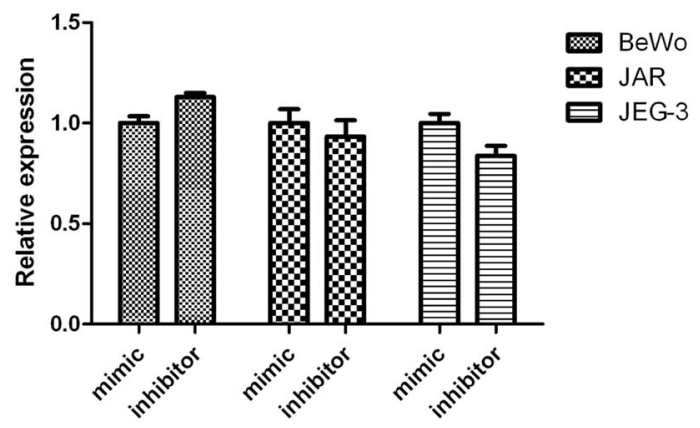

EFNA4

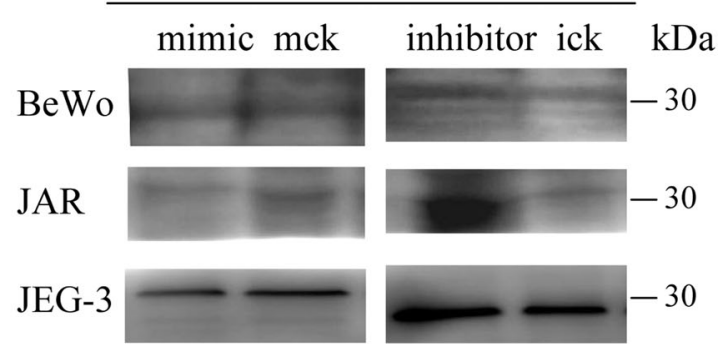

c

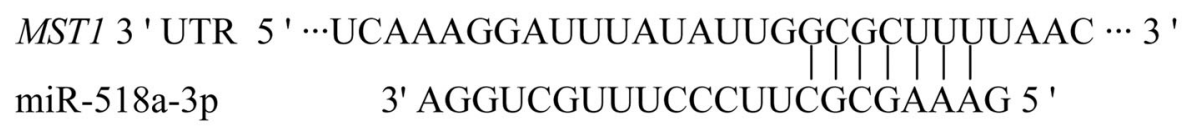

Mutant 3 ' UTR 5 ' $\cdots$ UCAAAgGAUUUAUAUUGACACGCGUAAC $\cdots 3$ '
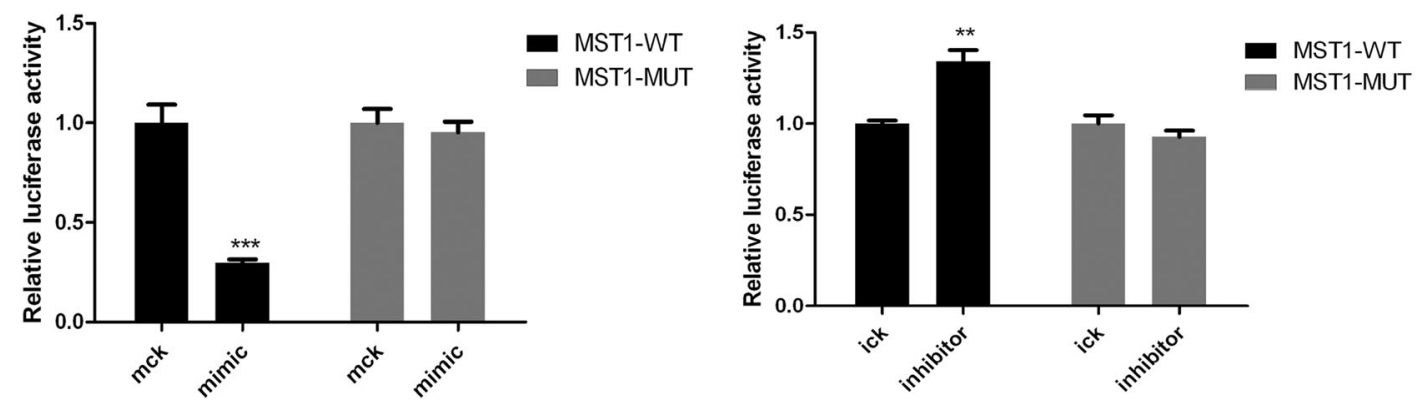

Fig. 6 Validation of miR-518a-3p potential target genes MST1 and EFNA4. a qRT-PCR quantification of the mRNA level of MST1 and EFNA4 genes. BeWo, JAR, and JEG-3 cells were transfected with miR-518a-3p mimic/inhibitor or controls. Cells were harvested at $36 \mathrm{~h}$ post transfection, and a-tubulin served as the endogenous reference gene. $\mathbf{b}$ Western blotting analysis of MST1 and EFNA4. Cells were harvested at $48 \mathrm{~h}$ post transfection, and a-tubulin was taken as the endogenous control. c Direct interaction of miR-518a-3p and MST1. Upper: bioinformatic predicted miR-518a-3p targeting site in 3'-UTR of MST1. Lower: luciferase activity assay. At $48 \mathrm{~h}$ post co-transfection with MST1-WT or MST1-MUT and miR-518a-3p mimic/ inhibitor or control mimic/inhibitor, luciferase activities were measured in HTR-8/SVneo cells. Renilla luciferase activities in corresponding controls were normalized to 1 . Mck stands for control mimic and ick stands for control inhibitor. $N=9,{ }^{* *} P<0.01,{ }^{* * *} P<0.001$ 
mimic/inhibitor. Similar results were obtained when miR518a-3p mimic/inhibitor co-transfected with MST1-WT or MST1-MUT (Fig. 6c).

MiR-371a-5p and miR-518a-3p targeted multiple pathways in tumorigenesis and metastasis

To identify the pathways and other differentially expressed genes (DEGs) that are potentially involved in miR-371a-5p and miR-518a-3p signaling, transcriptome sequencing was conducted in BeWo, JAR, and JEG-3 cells post miR-371a-5p and miR-518a-3p knockdown. A gene with a fragments per kilobase per million (FPKM) ratio larger than 2 or smaller than 0.5 and with the adjusted $P$ value $<0.05$ was considered to be a DEG. Totally, 453, 267, and 487 DEGs were identified in BeWo, JAR, and JEG-3 cells, respectively, after miR371a-5p knockdown. Among which, 237, 132, and 277 genes were upregulated, respectively. After miR-518a-3p knockdown, 503, 605, and 563 DEGs along with 229, 269 , and 191 upregulated ones were identified in BeWo, JAR, and JEG-3 cells, respectively. The top upregulated genes included many oncogenes or oncogenesis associated ones, such as $A K A P 2, M Y C$, and IGF2 for miR371a-5p, and SNX22, CDKL5, and PTTG1IP for miR518a-3p (Table 1). Kyoto Encyclopedia of Genes and Genomes (KEGG) enrichment analysis of the upregulated genes also showed that miR-371a-5p and miR518a-3p regulated diverse pathways related to tumorigenesis and metastasis, such as VEGF, TGF- $\beta$, and gap junction pathways for miR-371a-5p (Fig. 7a), and p53, cell cycle, and apoptosis pathways for miR-518a-3p (Fig. 7b).

\section{Discussion}

GTD is a range of pregnancy-related disorders. Some of the GTDs can further develop into life-threatening GTN. However, the molecular underpinnings driving GTD progression are largely unknown. MicroRNAs are regulators of genes generally at posttranscriptional level. Dysregulation of miRNAs is globally involved in tumorigenesis and cancer metastasis. Comparative analysis has revealed many differentially expressed miRNAs in cancers. In this study, we identified that miR-370-3p, $-371 a-5 p,-518 a-3 p,-519 d-3 p,-520 a-3 p$, and -934 were dysregulated in GTN. Although our microarray profiling data are still preliminary because of the small sample size, measurement of the six tissue sample-verified miRNAs would be helpful for the prognosis and clinical management of CHM patients. In this study, we took the $>5$-folds criterion for further verification in trophoblastic tissues. However, reports indicated that much smaller changes $\left(<2\right.$-folds) could also be significant ${ }^{23}$. Therefore, we cannot exclude other possible hallmark miRNAs in CHM progression.

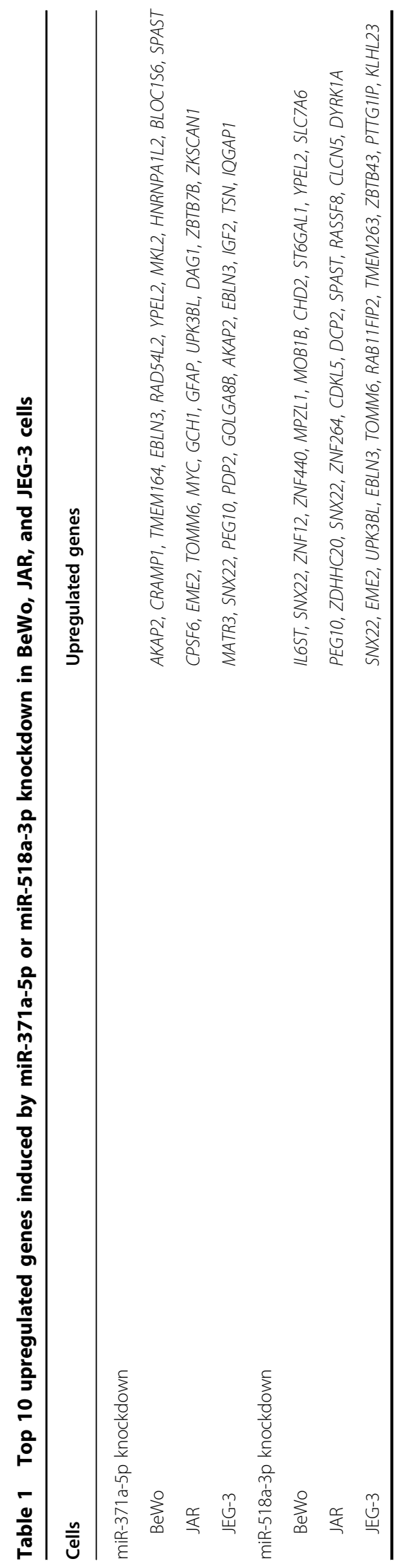




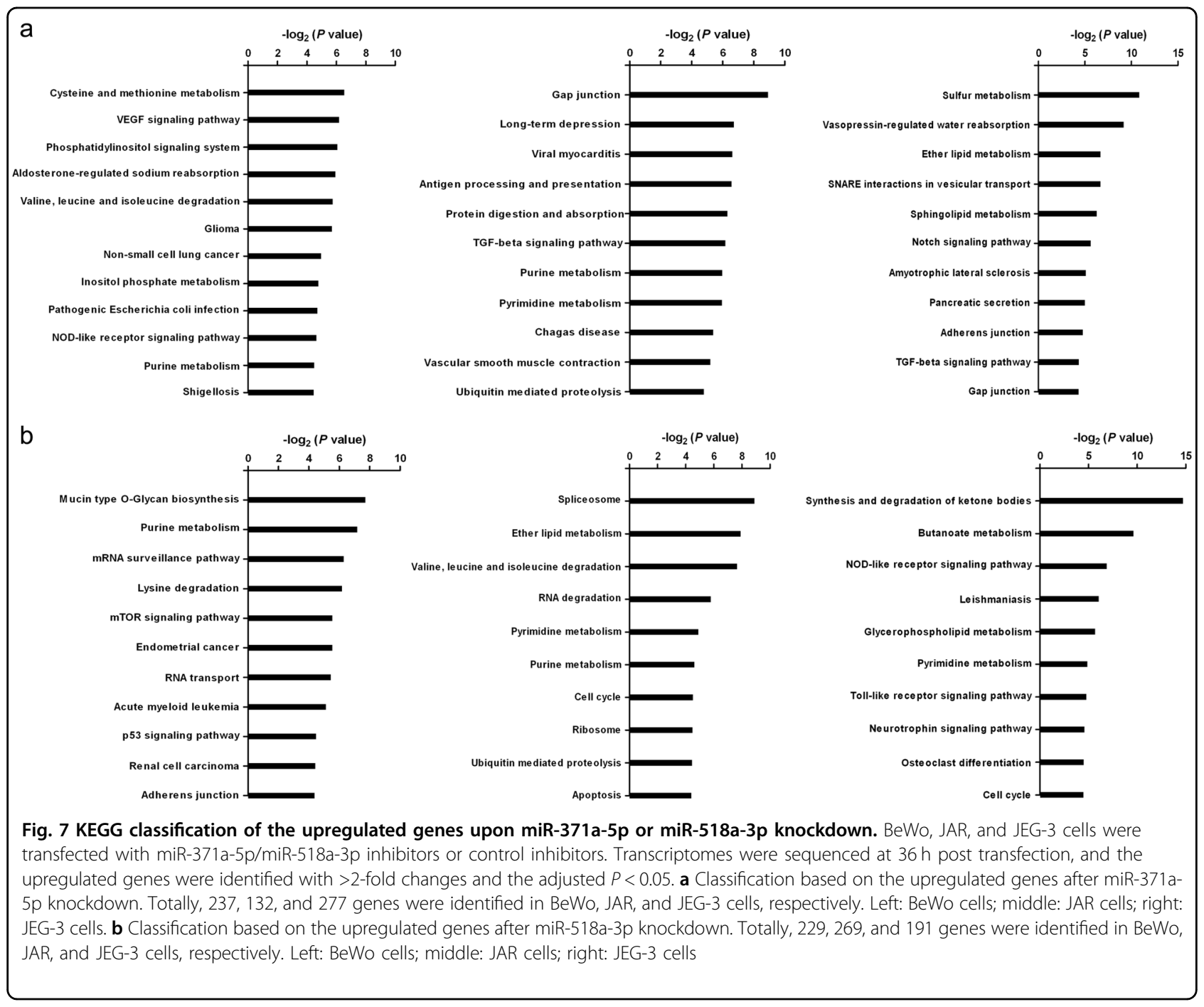

MiR-371a-5p is a member of the miR-371 373 cluster, which has been reported to be oncogenic, immune suppressive, and a stem cell signature ${ }^{24}$. However, studies about the functions and regulation networks of miR-371a$5 p$ are very limited. Upregulation of miR-371a-5p was identified in the development of hepatic fibrosis ${ }^{25}$. MiR$371 a-5 p$ dysregulation was also reported to be involved in the gemcitabine-resistance in pancreatic cancer cells ${ }^{26}$ and radioresistance in nasopharyngeal carcinoma cells ${ }^{27}$. MiR-518a-3p is a member of the primate-specific C19MC, which accounts for about $8 \%$ of all known human miRNA genes. C19MC plays important roles in cell differentiation and immunomodulation during pregnancy $^{28}$. Overexpression of miR-518a-3p has been detected in hepatocellular carcinoma, breast cancer, and Sezary syndrome. The metastatic foci in the lymph nodes of some tumors also showed upregulated miR-518a-3p compared with primary tumors ${ }^{29}$. Additionally, Port et al. reported that miR-518a-3p was upregulated in cisplatin- resistant germ cell tumor cell lines ${ }^{30}$. Nonetheless, reports about miR-518a-3p are diverse among different cancers. A study indicated that miR-518a-3p was downregulated in colorectal cancer cell lines and tissues with metastases ${ }^{31}$. $\mathrm{Xu}$ et al. also reported that miR-518a-3p was downregulated in chemo-resistant breast cancer cells ${ }^{32}$. Our study demonstrated that overexpressions of miR-371a-5p and miR-518a-3p promoted proliferation, migration, and invasion of choriocarcinoma cells. Meanwhile, knockdown of both miRNAs upregulated many genes in diverse pathways related to tumorigenesis and metastasis. Besides, many downregulated genes post miR-371a-5p and miR-518a-3p knockdown were associated with interferon responses. GO analysis of all the DEGs revealed that miR-371a-5p was mainly associated with phosphatidylinositol binding, TGF- $\beta$ response, interferon response, and apoptosis (Supplementary Figure S6a) while miR518a-3p was mainly associated with immune response (Supplementary Figure S6b). All these findings reinforced 
the pivotal "oncomiRNAs" and immunomodulator roles of miR-371a-5p and miR-518a-3p in the progression of GTD. Moreover, crosstalk between these two miRNAs cannot be excluded since a particular miRNA can modulate multiple pathways.

Generally, the regulatory function of miRNA depends on its target genes. However, studies about the target genes of miR-371a-5p and miR-518a-3p are few. One study reported that BAG3, a component of the chaperone-assisted autophagy pathway, was a direct target of miR-371a-5 $\mathrm{p}^{33}$. Another study proved that NIK, a regulator of NF- $\mathrm{KB}$, was a direct target of miR-518a-3p ${ }^{31}$. In the current study, we demonstrated that miR-371a-5p negatively regulated BCCIP, BNIP3L, and SOX2, and miR-518a-3p negatively regulated MST1 and EFNA4. Importantly, we proved that $B C C I P$ was a direct target of miR-371a-5p, and MST1 was a direct target of miR-518a$3 p$ for the first time. Therefore, miR-371a-5p and miR-518a-3p may drive the pathogenesis of GTN via regulating these tumor suppressors. However, in our transcriptome sequencing, only 4 genes, including PEG10, CYB561D1, TCF12, and SUOX, were commonly upregulated in BeWo, JAR, and JEG-3 cells after miR-371a-5p knockdown. Meanwhile, SNX22, HMGCS1, and NBPF12 were commonly upregulated after miR-518a-3p knockdown. Two factors may contribute to such a few commonly upregulated genes. One is possibly the genetic difference of those three cell lines. Our principal component analysis of the transcriptomes revealed that BeWo cells shared only about $80 \%$ and $83 \%$ similarities with JAR and JEG-3 cells, respectively, while JAR cells shared about 83\% similarity with JEG-3 cells. Another factor is possibly the intrinsic regulation mechanisms of miRNA. Numerous reports indicated that miRNA could repress many of their targets without detectable changes in mRNA levels $^{34}$. Thus, the $>2$-folds criteria in our data processing might be too stringent. The exact roles of those commonly identified genes in the progression of GTD still need to be clarified.

BCCIP, a BRCA2 and p $21^{\text {Waf1/Cip1 }}$ cofactor, is abundantly expressed in many tissues. It reaches its highest level at the $\mathrm{S} / \mathrm{G} 1$ border and remains high in the $\mathrm{S}$ phase $^{35}$. BCCIP is also required for the transactivation activity of $\mathrm{p} 53^{36}$. BNIP3L, a mitochondrial protein, has been revealed to be a proapoptotic transcriptional target of $\mathrm{p}^{3} 3^{37}$. Overexpresison of BCCIP can inhibit $\mathrm{S}$ to $\mathrm{G} 1$ progression, whereas downexpresison of BCCIP leads to chromosomal polyploidization and centrosome amplification ${ }^{38}$. Therefore, BCCIP may be involved in the miR-371a-5p-mediated proliferation of choriocarcinoma cells. SOX2 is a core transcription factor in the maintenance of the undifferentiated phenotype of stem cells. Our previous studies also have reported that SOX2 was downregulated in HM, choriocarcinoma tissues, and choriocarcinoma cells ${ }^{39}$.
Naturally, cytotrophoblasts own certain stem cell characteristics. Thus, downregulation of SOX2 in GTN might induce the alteration of the controlled normal self-renewal of cytotrophoblasts to facilitate the tumorigenesis.

MST1 is a highly conserved Ser/Thr kinase, acting as a tumor suppressor by restricting cell proliferation and survival. Loss or inactivation of MST1 has been found in many tumors, including colorectal cancer, hepatocellular carcinoma, and soft-tissue sarcoma ${ }^{40,41}$. Apart from the functions in apoptosis signaling, studies indicated that MST1 was also implicated in centrosome duplication and mitotic chromosome alignment ${ }^{42}$, which may explain our observation of the S-phase arrest by miR-518a-3p inhibitor. The effect of MST1 is reported to be opposite to that of $\mathrm{AKT}^{43}$, consistent with our previous report that the activated AKT signaling pathway was essential for the pathogenesis of $\mathrm{GTD}^{44}$. We thus assume that there may be a miR-518a-3p-MST1-AKT regulatory axis in the progression of GTN. Eph-ephrin system has been demonstrated to play important roles in migration of trophoblasts and in the initial step of embryo implantation. Nonetheless, studies about the function of EFNA4 in trophoblasts are few; only one report indicated that recombinant human ephrin A4 could promote the invasion of JEG-3 cells $^{45}$. In this study, the low expression of EFNA4 suggests that it may not play a major role in miR518a-3p-mediated metastasis of choriocarcinoma cells. Collectively, these findings suggest that miR-371a-5p and miR-518a-3p regulate the proliferation, migration, and invasion processes by interacting with diverse targets in trophoblasts, thereby affecting the pathogenesis of GTN.

In summary, we identified that miR-370-3p, $-371 a-5 p$, $-518 a-3 p,-519 d-3 p,-520 a-3 p$, and -934 were differentially expressed in GTN vs. CHM. We demonstrated that miR371a-5p and miR-518a-3p led to the aggressive characteristics of trophoblastic cells by regulating BCCIP, SOX2, and MST1. Our findings provide useful insight into the investigation of diagnostic biomarkers and therapeutic targets for GTN.

\section{Materials and methods}

\section{Tissue samples and ethics statement}

A total of 62 FFPE trophoblastic blocks, including 6 normal first-trimester placentas, 35 regressed $\mathrm{CHM}$ cases (CHMs), and 21 progressed CHM cases (GTN) after uterine evacuation, were retrieved from the bio-bank of International Peace Maternity and Child Health Hospital (IPMCH), Shanghai Jiao Tong University. Fresh trophoblastic tissues, including 6 cases of normal first-trimester placentas and 38 cases of CHMs (25 regressed ones), were also collected from the bio-bank of IPMCH. Every sample was diagnosed according to the morphological and clinical criteria ${ }^{1,2}$. Informed consent was obtained from each patient recruited. Ethical approval for the use of these 
tissues in this study was obtained from the IPMCH ethical review board.

\section{Cell culture}

Human choriocarcinoma cell lines BeWo, JAR, and JEG3 were obtained from Shanghai Cell Bank of Chinese Academy of Sciences (China), and were characterized by DNA fingerprinting and isoenzyme analysis. These cells were maintained in Dulbecco's modified Eagle's medium (DMEM, Invitrogen, NY, USA) supplemented with $10 \%$ fetal bovine serum (FBS, Invitrogen) at $37^{\circ} \mathrm{C}$. HTR-8/ SVneo, an immortalized normal trophoblastic cell line, was cultured in RPMI 1640 medium (Invitrogen) supplemented with $10 \%$ FBS. Villous primary human trophoblasts (PHT) were isolated from first-trimester placentas as described previously $^{46}$ and were cultured in DMEM/F12 medium (Invitrogen) supplemented with 15\% FBS.

\section{miRNA array}

Total RNA was extracted from fresh trophoblastic tissues with Trizol reagent (Invitrogen). RNA concentration was determined by Nanodrop 2000c (Thermo Fisher Scientific, Waltham, MA, USA). RNA integrity was evaluated by using an Agilent 2100 Bioanalyzer (Agilent, Santa Clara, CA, USA). MiRNA was then purified with mirVana $^{\mathrm{TM}}$ miRNA Isolation Kit (Thermo Fisher Scientific). After Poly(A) tailing and biotin labeling, hybridization was conducted on Affymetrix GeneChip miRNA 4.0. Data were obtained and analyzed with AGCC software (Affymetrix, Santa Clara, CA, USA).

\section{Quantitative RT-PCR}

Cells were washed three times with corresponding serum-free culture medium before harvesting. Total RNA was extracted from fresh trophoblastic tissues or cells with Trizol reagent. Extraction of miRNA from FFPE tissues was carried out with miRNeasy FFPE Kit (Qiagen, Hilden, Germany). For mRNA analysis, cDNA synthesis was carried out by using the PrimeScript 1st Strand cDNA Synthesis Kit (Takara, Dalian, China) and qRT-PCR was carried out by using the SYBR qRT-PCR Kit (Takara). For miRNA quantification, miRNA cDNA 1st Strand Kit and miRNA qPCR Assay Kit (CWBIO, Beijing, China) were used along with gene-specific primers. All qRT-PCR reactions were performed in triplicate on an ABI StepOneplus system (Applied Biosystems, Foster, CA, USA). $\alpha$-tubulin was used as the endogenous reference gene for mRNA analysis. RNU6 was used as the reference gene for miRNA analysis. Relative mRNA or miRNA level was calculated by using the $2^{-\Delta \Delta C t}$ method.

\section{ISH and fluorescence in situ hybridization (FISH)}

ISH and FISH were carried out on 6 - $\mu$ m-thick FFPE sections. Two double-digoxigenin (DIG)-labeled oligonucleotide probes, complementary to mature miR371a-5p (Exon Biotechnology, Guangzhou, China) and miR-518a-3p (Genscript, Nanjing, China), respectively, were synthesized. An RNU6-specific probe was used as the positive control. After deparaffinization, hydration, and proteinase $\mathrm{K}$ digestion, $40 \mathrm{pmol}$ probe was applied for each section. Hybridization was carried out at $55^{\circ} \mathrm{C}$ for 1 $\mathrm{h}$ in a Leica hybridizer (Wetzlar, Germany), and then washed in a decreasing gradient of SSC buffers at $55^{\circ} \mathrm{C}$. Next, sections were incubated for $15 \mathrm{~min}$ at $37^{\circ} \mathrm{C}$ with blocking solution, and then with sheep anti-DIG-AP antibody (1:200 diluted, Boster, Wuhan, China) for $1 \mathrm{~h}$ at $37^{\circ} \mathrm{C}$. Finally, sections were visualized with chromogene NBT/BCIP (Boster) and nuclei were stained with nuclear fast red (Boster). All sections were scored by three pathologists independently and blindly, and dominant staining intensity was scored as follows: negative (-), weak $(+)$, intermediate $(++)$, and strong $(+++)$. For FISH, the sheep anti-DIG-AP antibody was replaced with sheep anti-DIG-FITC antibody (1:100 diluted, Boster) and nuclei were stained with DAPI (Boster). Sections were analyzed under a Leica microscope with LAS v4.2 software.

\section{Transfection of the miRNA mimic and inhibitor}

MiRNA mimic/inhibitor and the control mimic/inhibitor were synthesized by RiboBio Company (Guangzhou, China). MiRNA mimics are double-stranded RNA molecules that mimic endogenous mature miRNA whereas miRNA inhibitors are single-stranded nucleic acids that specifically bind and inhibit endogenous miRNA ${ }^{47}$. Choriocarcinoma cells were plated in 24-well plates the day before transfection and grown to $70-80 \%$ confluence. Cells were then transfected with $150 \mathrm{nM}$ inhibitor or 100 $\mathrm{nM}$ mimic by using Lipofectamine 3000 (Invitrogen) according to the manufacturer's instructions.

\section{Cell proliferation assay}

BeWo, JAR, and JEG-3 cells were seeded in 96-well plates at $1 \times 10^{4}$ cells per well density the day before transfection. Twenty-four hours post transfection with 30 $\mathrm{nM}$ mimic or $40 \mathrm{nM}$ inhibitor, cell viability was measured every 12 or $24 \mathrm{~h}$ with Cell Counting Kit 8 reagent (DOJINDO, Kumamoto, Japan). Optical density was read at $450 \mathrm{~nm}$ using a universal microplate reader (Bio-Tek, Winooski, VT, USA).

\section{Cell cycle analysis}

About $1 \times 10^{6}$ cells were collected at $48 \mathrm{~h}$ post transfection. After washing twice with ice-cold PBS, cells were fixed in precooled $70 \%$ ethanol at $-20{ }^{\circ} \mathrm{C}$ overnight. Before analysis by flow cytometry, cells were washed three times with PBS, resuspended in $500 \mu \mathrm{l}$ propidium iodide/ RNase staining solution (BD Biosciences, San Jose, CA, 
USA), and incubated in the dark for $30 \mathrm{~min}$ at $37^{\circ} \mathrm{C}$. The percentage of cells in each phase of cell cycle was calculated by using the Multicycle Program (Beckman Coulter, Brea, CA, USA).

\section{Cell migration and invasion assays}

Cell migration was detected by using transwell assay. Briefly, at $24 \mathrm{~h}$ post transfection, cells were pretreated with mitomycin C (Selleck, Shanghai, China) for $2 \mathrm{~h}$. Then, $5 \times$ $10^{4}$ cells suspended in $100 \mu \mathrm{l}$ serum-free DMEM were seeded into the transwell insert with 8 - $\mu$ m-diameter pore membrane (Corning, Shanghai, China). As a chemoattractant, $500 \mu \mathrm{l}$ DMEM medium containing 15\% FBS was added into the lower chamber. After incubation at $37^{\circ} \mathrm{C}$ for $36 \mathrm{~h}$, non-migrated cells on the upper surface of the membrane were removed with cotton swabs. Cells that attached to the undersurface of the membrane were fixed with $4 \%$ paraformaldehyde for $30 \mathrm{~min}$, and then stained with $0.1 \%$ crystal violet for $15 \mathrm{~min}$. For invasion assay, the upper chamber was coated with $100 \mu$ l Matrigel (BD Biosciences) before incubation. Then, $1 \times 10^{5}$ cells were seeded and incubated for $48 \mathrm{~h}$ at $37^{\circ} \mathrm{C}$. Invaded cells were fixed and stained following the same protocol as the migration assay. Cells were counted under a light microscope (Olympus, Tokyo, Japan) at $100 \times$ magnification, and five randomly selected fields were scored per well. Mean cell number of the five fields was calculated to be the number of migrated or invaded cells for that well. Both assays were carried out in triplicate.

\section{Western blotting}

Cells were harvested at $48 \mathrm{~h}$ post transfection, and lysed in SDS lysis buffer (Beyotime, Beijing, China) with fresh addition of $1 \%$ protease inhibitor cocktail (Sigma-Aldrich, Darmstadt, Germany) and $1 \mathrm{mM}$ PMSF (Sigma-Aldrich). Protein concentrations were determined by the Bradford method with the BCA Protein Assay kit (Thermo Fisher Scientific). Equal amount of proteins $(30 \mu \mathrm{g})$ were resolved in $10 \%$ SDS-PAGE and then transferred to PVDF membranes (Millipore, Billerica, MA, USA). After blocking, PVDF membranes were washed and incubated with different primary antibodies [1:1000 diluted mouse antiBCCIP (08269, Sigma-Aldrich), rabbit anti-BNIP3L (12,396, Cell signaling technology, Danvers, MA, USA), rabbit anti-SOX2 (3579, Cell signaling technology), goat anti-MST1 (AF4949, R\&D systems, Minneapolis, MN, USA), goat anti-EFNA4 (AF369, R\&D systems) antibodies, 1:5000 diluted rabbit anti- $\alpha$-tubulin monoclonal antibody (2125, Cell signaling technology)] at $4{ }^{\circ} \mathrm{C}$ overnight. After re-warming for $30 \mathrm{~min}$ at room temperature and three times washing, membranes were incubated with HRP-linked goat anti-rabbit, goat anti-mouse, or rabbit anti-goat IgG (1:5000 diluted, Jackson ImmunoResearch, West Grove, PA, USA) for $1 \mathrm{~h}$ at $37^{\circ} \mathrm{C}$. Protein bands were developed by using the ECL kit (Tiangen Biotech, Beijing, China), and images were captured with Amersham Imager 600 (GE Healthcare, Chicago, IL, USA).

\section{Luciferase reporter assays}

Wild-type or mutated 3 '-UTR regions of BCCIP and $M S T 1$ were cloned into the psiCHECK-2 vector (Promega, Madison, WI, USA) and nominated as BCCIP-WT, BCCIP-MUT, MST1-WT, and MST1-MUT, respectively. Then, HTR-8/SVneo cells were seeded in 24-well plates the day before transfection and grown to $70 \%$ confluence. Plasmids (400 ng) were co-transfected with corresponding miRNA mimic/inhibitor or control mimic/ inhibitor $(100 \mathrm{nM})$ by using Lipofectamine 3000 reagent. At $48 \mathrm{~h}$ post transfection, cells were lysed and luciferase activity was measured by using the Dual-Luciferase Reporter Assay System (Promega). Renilla luciferase signal was normalized to firefly luciferase signal. Each assay was performed in triplicates and three parallel wells were set for each time.

\section{Transcriptome sequencing}

BeWo, JAR, and JEG-3 cells were transfected with miR371a-5p or miR-518a-3p inhibitors or control inhibitor. At $36 \mathrm{~h}$ post transfection, cells were washed three times with PBS. Total RNA was extracted with the RNeasy Mini Kit (Qiagen). RNA concentration was determined by Nanodrop 2000c. RNA integrity was evaluated by using an Agilent 2100 Bioanalyzer. Poly(A) RNA were then purified with oligo(dT) beads and used for cDNA library generation and paired-end transcriptome sequencing (ShanghaiBio, Shanghai, China). Sequencing was performed using an Illumina HiSeq 2500 (San Diego, CA, USA) with more than $6 \mathrm{G}$ bases throughput for every sample. Reads were aligned to the human genome (http://www.ensembl.org/ Homo_sapiens/Info/Index) using HISAT2 ${ }^{48}$. Data were normalized as FPKM. DEGs were identified with an adjusted $P$ value $<0.05$ and a fold change $>2$. GO (http:// www.geneontology.org/) and KEEG (http://www.genome. $\mathrm{jp} / \mathrm{kegg} /$ ) pathway enrichment analyses were then performed based on DEGs or upregulated genes.

\section{Statistical analysis}

All experiments were performed at least in triplicates. Data were presented by mean \pm SD where applicable. Differences were evaluated by using Mann-Whitney $U$ test, $\chi^{2}$ test, and $t$-test for two-group comparisons, while one-way ANOVA, Kruskal-Wallis, and $\chi^{2}$ tests for multiple comparisons. Statistical analyses were performed using R 3.1.3 (Lucent Technologies, Murray Hill, NJ, USA), SPSS 14.0 (IBM, Armonk, NY, USA), or GraphPad Prism 5 (GraphPad Software, La Jolla, CA, USA) software packages. The probability of $P<0.05$ was considered to be statistically significant. 


\section{Acknowledgements}

We thank Dr. Charles H. Graham (Queen's University at Kingston) for the kind gift of HTR8/SVneo cell line. We are particularly grateful to Prof. Yoel Sadovsky (University of Pittsburgh) for critical experiment design and invaluable suggestions. This work was supported by National Natural Science Foundation of China (grant numbers: 81501255, 81072140), Shanghai Municipal Science and Technology Commission (grant number: 17ZR1433000), and Shanghai Municipal Health and Family Planning Commission (grant number: 201640033).

\section{Author details}

'Departments of Pathology and Bio-Bank, International Peace Maternity and Child Health Hospital, Shanghai Jiao Tong University School of Medicine, Shanghai 200030, China. Institute of Embryo-Fetal Original Adult Disease Shanghai Jiao Tong University School of Medicine, Shanghai 200030, China. ${ }^{3}$ Department of Obstetrics, International Peace Maternity and Child Health Hospital, Shanghai Jiao Tong University School of Medicine, Shanghai 200030 China. ${ }^{4}$ Department of Ultrasound in Medicine, Shanghai Jiao Tong University Sixth People's Hospital, Shanghai 200233, China

\section{Competing interests}

The authors declare that they have no competing financial interests.

\section{Publisher's note}

Springer Nature remains neutral with regard to jurisdictional claims in published maps and institutional affiliations.

\section{Supplementary information}

The online version of this article (https://doi.org/10.1038/s41419-017-0108-2) contains supplementary material.

\section{Received: 13 July 2017 Revised: 20 October 2017 Accepted: 26 October} 2017

Published online: 24 January 2018

\section{References}

1. Ngan, H. Y. S. The practicability of FIGO 2000 staging for gestational trophoblastic neoplasia. Int. J. Gynecol. Cancer 14, 202-205 (2004).

2. Kurman, R. J., Carcangiu, M. L., Herrington, C. S. \& Young, R. H. (eds) WHO Classification of Tumours of Female Reproductive Organ 4th edn (IARC Press, Lyon, France, 2014).

3. Szulman, A. E. in Gestational Trophoblastic Disease (eds Szulman, A. E. \& Buchsbaum, H. J.) 37-44 (Springer New York, New York, USA, 1987).

4. Steigrad, S. J. Epidemiology of gestational trophoblastic diseases. Best Pract. Res. Clin. Obstet. Gynaecol. 17, 837-847 (2003).

5. Ngu, S.F. \& Chan, K. K. L. Management of chemoresistant and quiescent gestational trophoblastic disease. Curr. Obstet. Gynecol. Rep. 3, 84-90 (2014).

6. Baasanjav, B. et al. The risk of post-molar gestational trophoblastic neoplasia is higher in heterozygous than in homozygous complete hydatidiform moles. Hum. Reprod. 25, 1183-1191 (2010).

7. Neubauer, N. L., Strohl, A. E., Schink, J. C. \& Lurain, J. R. Fatal gestational trophoblastic neoplasia: an analysis of treatment failures at the Brewer Trophoblastic Disease Center from 1979-2012 compared to 1962-1978. Gynecol. Oncol. 138, 339-342 (2015).

8. Hobert, O. Gene regulation by transcription factors and microRNAs. Science 319, 1785-1786 (2008)

9. Calin, G. A. \& Croce, C. M. MicroRNA signatures in human cancers. Nat. Rev. Cancer 6, 857-866 (2006).

10. Iorio, M. V. et al. MicroRNA gene expression deregulation in human breast cancer. Cancer Res. 65, 7065-7070 (2005).

11. Lee, E. J. et al. Expression profiling identifies microRNA signature in pancreatic cancer. Int. J. Cancer 120, 1046-1054 (2007).

12. Yanaihara, N. et al. Unique microRNA molecular profiles in lung cancer diagnosis and prognosis. Cancer Cell 9, 189-198 (2006).

13. Piletic, K. \& Kunej, T. MicroRNA epigenetic signatures in human disease. Arch. Toxicol. 90, 2405-2419 (2016)
14. Iorio, M. V. \& Croce, C. M. MicroRNA dysregulation in cancer: diagnostics, monitoring and therapeutics. A comprehensive review. EMBO Mol. Med. 4 143-159 (2012).

15. Iorio, M. V. \& Croce, C. M. microRNA involvement in human cancer. Carcinogenesis 33, 1126-1133 (2012).

16. Rippe, V. et al. Activation of the two microRNA clusters C19MC and miR371-3 does not play prominent role in thyroid cancer. Mol. Cytogenet. 5, 40 (2012).

17. $\mathrm{Na}, \mathrm{Q}$., Wang, D. \& Song, W. Underexpression of 4 placenta-associated microRNAs in complete hydatidiform moles. Int. J. Gynecol. Cancer 22, 1075-1080 (2012)

18. Hasegawa, Y., Miura, K, Furuya, K, Yoshiura K-i \& Masuzaki, H. Identification of complete hydatidiform mole pregnancy-associated microRNAs in plasma. Clin. Chem. 59, 1410-1412 (2013).

19. Miura, K. et al. Identification of pregnancy-associated microRNAs in maternal plasma. Clin. Chem. 56, 1767-1771 (2010).

20. Chao, A. et al. Decreased expression of microRNA-199b increases protein levels of SET (protein phosphatase 2A inhibitor) in human choriocarcinoma. Cancer Lett. 291, 99-107 (2010).

21. Wang, Y.-X., Zhao, J.-R., Xu, Y.-Y., Wu, W.-B. \& Zhang, H.-J. MiR-21 is overexpressed in hydatidiform mole tissues and promotes proliferation, migration, and invasion in choriocarcinoma cells. Int. J. Gynecol. Cancer 27, 364-374 (2017).

22. Luo, L. et al. MicroRNA-378a-5p promotes trophoblast cell survival, migration and invasion by targeting Nodal. J. Cell Sci. 125, 3124-3132 (2012).

23. Sadovsky, Y., Mouillet, J.-F., Ouyang, Y., Bayer, A. \& Coyne, C. B. The function of trophomiRs and other microRNAs in the human placenta. Cold Spring Harb. Perspect. Med. 5, a023036 (2015).

24. Murray, M. J. et al. Identification of microRNAs from the miR-371 373 and miR-302 clusters as potential serum biomarkers of malignant germ cell tumors. Am. J. Clin. Pathol. 135, 119-125 (2011).

25. Zhang, Q. et al. Analysis of the differential expression of circulating microRNAs during the progression of hepatic fibrosis in patients with chronic hepatitis B virus infection. Mol. Med. Rep. 12, 5647-5654 (2015).

26. Shen, $Y$. et al. Identifying microRNA-mRNA regulatory network in gemcitabineresistant cells derived from human pancreatic cancer cells. Tumour Biol. 36, 4525-4534 (2015).

27. Li, G. et al. Genome-wide analyses of radioresistance-associated miRNA expression profile in nasopharyngeal carcinoma using next generation deep sequencing. PLOS ONE 8, e84486 (2013).

28. Rippe, $\mathrm{V}$. et al. The two stem cell microRNA gene clusters C19MC and miR371-3 are activated by specific chromosomal rearrangements in a subgroup of thyroid adenomas. PLoS ONE 5, e9485 (2010).

29. Baffa, R. et al. MicroRNA expression profiling of human metastatic cancers identifies cancer gene targets. J. Pathol. 219, 214-221 (2009).

30. Port, M. et al. MicroRNA expression in cisplatin resistant germ cell tumor cell lines. Mol. Cancer 10, 52 (2011).

31. Qu, L. L., He, L., Zhao, X. \& Xu, W. Downregulation of miR-518a-3p activates the NIK-dependent NF-kappaB pathway in colorectal cancer. Int. J. Mol. Med. 35 , 1266-1272 (2015).

32. $\mathrm{Xu}, \mathrm{P}$. et al. Identification and characterization of microRNAs expressed in human breast cancer chemo-resistant MCF-7/Adr cells by Solexa deepsequencing technology. Biomed. Pharmacother. 75, 173-178 (2015).

33. d'Avenia, M. et al. A novel miR-371a-5p-mediated pathway, leading to BAG3 upregulation in cardiomyocytes in response to epinephrine, is lost in Takotsubo cardiomyopathy. Cell Death Dis. 6, e1948 (2015).

34. Baek, D. et al. The impact of microRNAs on protein output. Nature $\mathbf{4 5 5}, \mathbf{6 4 - 7 1}$ (2008).

35. Meng, X., Liu, J. \& Shen, Z. Genomic structure of the human BCCIP gene and its expression in cancer. Gene 302, 139-146 (2003).

36. Meng, X., Yue, J., Liu, Z. \& Shen, Z. Abrogation of the transactivation activity of p53 by BCCIP down-regulation. J. Biol. Chem. 282, 1570-1576 (2007).

37. Fei, P. et al. Bnip3L is induced by p53 under hypoxia, and its knockdown promotes tumor growth. Cancer Cell 6, 597-609 (2004).

38. Meng, X., Fan, J. \& Shen, Z. Roles of BCCIP in chromosome stability and cytokinesis. Oncogene 26, 6253-6260 (2007).

39. Li, A. S. M. et al. Hypermethylation of SOX2 gene in hydatidiform mole and choriocarcinoma. Reprod. Sci. 15, 735-744 (2008).

40. Minoo, P. et al. Prognostic significance of mammalian sterile20-like kinase 1 in colorectal cancer. Modern Pathol. 20, 331-338 (2007). 
41. Zhou, D. et al. Mst1 and Mst2 protein kinases restrain intestinal stem cell proliferation and colonic tumorigenesis by inhibition of Yes-associated protein (Yap) overabundance. Proc. Natl Acad. Sci. USA 108, E1312-E1320 (2011).

42. Cornils, H., Kohler, R. S., Hergovich, A. \& Hemmings, B. A. Downstream of human NDR kinases: impacting on c-myc and p21 protein stability to control cell cycle progression. Cell Cycle 10, 1897-1904 (2011).

43. Ling, P., Lu, T. J., Yuan, C. J. \& Lai, M. D. Biosignaling of mammalian Ste20related kinases. Cell Signal 20, 1237-1247 (2008).

44. Wang, Y. X., Zhang, H. J., Liu, Y., Wu, W. B. \& Xu, Y. Y. The activation of PI3K/PKB signaling in pathogenesis of gestational trophoblastic disease. Prog. Obstet. Gynecol. 24, 22-29 (2015).
45. Fujiwara, $H$. et al. Eph-Ephrin A system regulates human choriocarcinoma-derived JEG-3 cell invasion. Int. J. Gynecol. Cancer 23 576-582 (2013).

46. Wu, W. et al. Dysregulated activation of c-Src in gestational trophoblastic disease contributes to its aggressive progression. Placenta $\mathbf{3 5}$, 824-830 (2014)

47. Bader, A. G., Brown, D., Stoudemire, J. \& Lammers, P. Developing therapeutic microRNAs for cancer. Gene Ther. 18, 1121-1126 (2011).

48. Kim, D., Langmead, B. \& Salzberg, S. L. HISAT: a fast spliced aligner with low memory requirements. Nat. Methods 12, 357-360 (2015). 